\title{
Direct evidence of cognitive control without perceptual awareness
}

\author{
Brenda Ocampo • Shahd Al-Janabi • \\ Matthew Finkbeiner
}

Published online: 18 November 2014

(C) Psychonomic Society, Inc. 2014

\begin{abstract}
A central question within the domain of human cognition is whether or not the ability to replace a current action with a new one (i.e., cognitive control) depends on a conscious appreciation of the environmental change that necessitates the new behavior. Specifically, it is not yet known if non-consciously perceived stimuli can trigger the modification of a currently ongoing action. We show for the first time that individuals are able to use non-consciously perceived information to modify the course and outcome of an ongoing action. Participants were presented with a masked (i.e., subliminal) 'stop' or 'go-on' prime stimulus whilst performing a routine reach-to-touch action. Despite being invisible to participants, the stop primes produced more hesitations mid-flight and more movement reversals than the go-on primes. This new evidence directly establishes that cognitive control (i.e., the ability to modify a currently ongoing action) does not depend on a conscious appreciation of the environmental trigger.
\end{abstract}

Keywords Cognitive control and automaticity · Inhibition . Priming $\cdot$ Visual awareness

Cognitive control is needed when an ongoing routine behavior (e.g., driving a car) is unexpectedly interrupted by information

B. Ocampo $(\bowtie)$

School of Psychology, Australian Catholic University,

Brisbane 4014, QLD, Australia

e-mail: brenda.ocampo@mindsci.net

S. Al-Janabi

Department of Psychology, University of Wisconsin, Milwaukee, WI, USA

M. Finkbeiner

Perception in Action Research Centre \& Department of Cognitive

Science, Macquarie University, Sydney, NSW, Australia (e.g., a pedestrian crossing the street) that requires the execution of a new, adaptive behavior (e.g., slamming on the brakes; c.f. van Gaal, Ridderinkhof, Scholte, \& Lamme, 2010). This example illustrates a hallmark of cognitive control: our ability to adjust presently-unfolding actions in response to new and unexpected information. Until recently, cognitive control was thought to depend upon conscious awareness such that only consciously perceived events could trigger the active suppression and/or replacement of an already-initiated action or behavior (Baars, 2002; Dehaene \& Naccache, 2001). Contrary to this view, however, several recent findings suggest that consciousness and cognitive control may be dissociable (Hughes, Velmans, \& De Fockert, 2009; van Gaal, Lamme, \& Ridderinkhof, 2010; van Gaal, Ridderinkhof, et al., 2010; van Gaal, Ridderinkhof, van den Wildenberg, \& Lamme, 2009).

In one of the earliest reports, Hughes and colleagues (2009) used masked primes to influence responses in a go/no-go paradigm. Their participants were instructed to execute a speeded response following the onset of a go target (e.g., a right-pointing arrow), but inhibit the response following onset of the no-go target (e.g., a left-pointing arrow). These target stimuli were preceded by masked go/no-go primes (also left/right pointing arrows). Although not consciously detected, participants' responses were nevertheless influenced by the primes. Specifically, reaction times (RTs) to go targets were faster when preceded by a go prime and slower when preceded by a no-go prime. Prime stimuli also modulated two event-related potential components associated with response inhibition, the N2 and the P3. Normally elicited by no-go targets that require an inhibitory response, both components were significantly reduced when targets were preceded by no-go primes. The findings of Hughes and colleagues (2009) thus reflect the non-conscious influence over the planning of an upcoming response.

Using a modified version of the stop-signal task, van Gaal and colleagues (2009) asked participants to make a speeded 
response to a go signal except when it was followed by a stop signal. The visibility of the stop signal was manipulated using a masking procedure that caused some of the stop signals to be subliminal. Interestingly, participants' responses were slowed by stop signals even though they could not be consciously perceived. Together, these studies convincingly demonstrate that non-consciously perceived stimuli can lead to the partial suppression and, in some cases, the successful vetoing of a planned, but not yet executed, action.

While these findings have been tremendously successful in challenging the long-held view that cognitive control depends on consciousness, it remains to be seen whether an arbitrarily defined stimulus that is presented non-consciously can trigger modulations of an action that is already being executed. For example, while it is already well established that individuals can adjust ongoing reach-to-touch movements following nonconsciously perceived shifts in the spatial position of the target (see Gaveau et al., 2014), it remains to be seen whether such adjustments can be made based on a participant's interpretation of a non-consciously perceived target. Thus, for the present study we chose to use a "choice-determined response" paradigm (Finkbeiner, Coltheart \& Coltheart, 2013), where the correct response is determined by the subject's interpretation of the stimulus, not its spatial position. The aim of the study is clear: to establish whether a participant's ongoing reaching response can be influenced by their interpretation of a non-consciously perceived and arbitrarily defined stimulus.

More specifically, we asked whether an automatized routine action that is already underway can be interrupted following the presentation of a subliminal stop signal. To do this, participants were required to make a fast reach-to-touch movement towards a response region $50 \mathrm{~cm}$ in front of them; this constituted the 'default' action. Participants initiated their reaching movement in synchrony with an auditory tone. Critically, we presented the visual "go-on" or "stop" target stimulus (the letters 'A' or 'B') after they had commenced their movement. Participants were instructed to continue their default action in the event of a go-on target ( $80 \%$ of all trials), and to terminate their action mid-flight and return to the start position in the event of a stop target ( $20 \%$ of all trials). Unbeknownst to the participants, the targets were preceded by a masked prime stimulus (the letter 'a' or 'b'), which were $50 \%$ congruent (e.g., go-on targets preceded by go-on primes) and $50 \%$ incongruent (i.e., go-on targets preceded by stop primes). We reasoned that if a presently-unfolding action is subject to non-conscious cognitive control, we should see a systematic influence of the masked prime stimulus on the kinematic properties of the default reaching movement. To anticipate our results, we find (1) that subjects failed to terminate incongruent stop target trials more often and (2) that incongruent go-on target trials were interrupted more often and took longer to complete.

\section{Experiment 1}

Method

\section{Participants}

For the first experiment, 16 volunteers (4 males, aged 18 30 years) were recruited from the undergraduate subject pool at Macquarie University. For the second experiment, 16 new volunteers were recruited from the same pool ( 3 males, aged 18-28 years). All gave written informed consent to participate in the experiment, which was approved by the Human Research Ethics Committee of Macquarie University. All participants were right-handed, had normal or corrected-tonormal vision, and were naïve to the purpose of the study.

\section{Stimuli and procedure}

Each trial began with the word "Start" in the center of the screen. At this point, participants placed their right index finger on the home button, which was aligned with the body midline $1 \mathrm{~cm}$ from the table edge (Fig. 1). This action prompted the onset of the forward mask '\#', which appeared at fixation. Participants were instructed to initiate the default reaching movement within a 300-ms-wide response window surrounding an auditory go signal (the final beep in a sequence of three beeps). The response window opened $100 \mathrm{~ms}$ before the go signal and closed $300 \mathrm{~ms}$ later. If a movement was not initiated within the response window, the trial was terminated with a buzz and the appropriate visual feedback (e.g. "Too Early!" or "Too Late!") was presented.

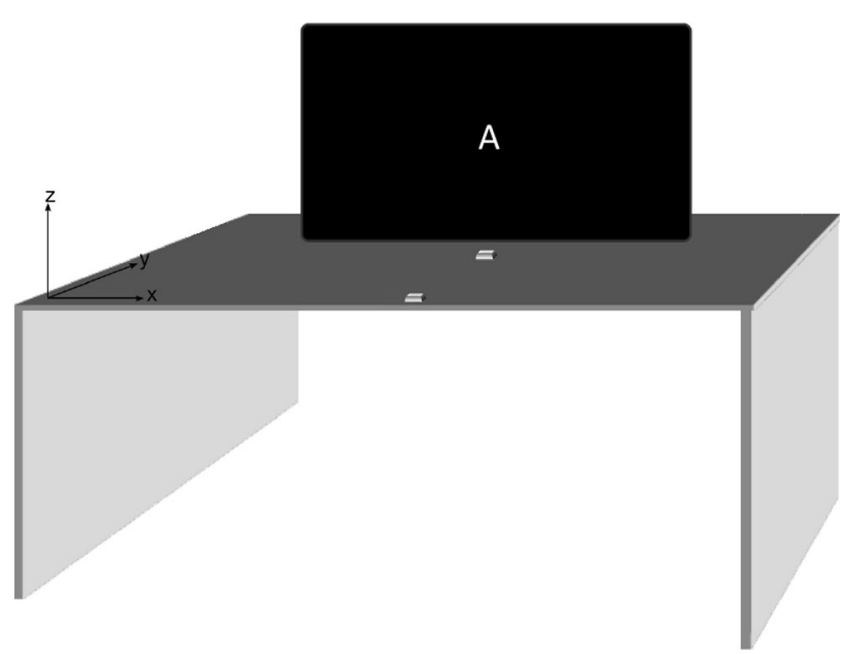

Fig. 1 Experimental set-up during Experiments 1 and 2. Participants sat at a desk with an LED monitor and two response buttons. They placed their right index finger on the home button (white square closest to table edge) to initiate the trial, and responded in synchrony with the last of three auditory tones by reaching out to touch the furthermost response button (closest to the monitor) 
Immediately following release of the home button, one of two target letters (' $A$ ' or ' $B$ ') would then appear in the center of the screen. One letter was the "stop target" and the other was the "go-on target" (counterbalanced). Eighty percent of the targets were go-on targets, thereby leading participants to expect to perform an uninterrupted movement in the forward direction. As mentioned above, the targets were preceded by a masked prime stimulus ('a' or 'b'). The primes were presented for $30 \mathrm{~ms}$ and immediately followed by the target, which served as a backward mask. To ensure participants focused their attention on the location of the primetarget sequence, the targets were presented for only $100 \mathrm{~ms}$ and then backward masked with an ampersand ' $\&$ ' that remained on the screen until the correct response was made.

To discourage participants from producing partial movements, whereby they paused midflight until they knew the trial type (go-on or stop), we required participants to maintain a continuous forward movement over the first 50 recorded samples ( 208 ms). Reaching movements that failed to do this were terminated with a buzz and visual feedback. Each participant completed a total of 350 experimental trials with $80 \%$ being go-on trials and $20 \%$ being stop trials. Again, half of the prime-target pairs were congruent and half were incongruent. In Experiment 2, the design was changed slightly to include a neutral prime (' $x$ '), which never appeared as a target. A total of 525 experimental trials were completed ( $80 \%$ go-on trials $/ 20 \%$ stop trials). Both go-on and stop targets were preceded with equal probability by either a go-on prime, a stop prime, or a neutral prime.

\section{Apparatus}

A Samsung S27SA950 LED monitor was used at a resolution of $1920 \times 1080(120 \mathrm{~Hz})$, controlled by a Dell Optiplex GX990 running 64-bit Windows. Stimulus presentation was controlled using Presentation software (Neurobehavioral Systems), and custom software was written to interface the stimulus display with a motion capture device. A Polhemus Liberty system was used to record motion data sampling at $240 \mathrm{~Hz}$, via a small magnetic sensor attached to the tip of the right index finger. In Experiment 2, an Optotrak Certus device was used to sample the position of a small light-emitting diode fixed to the tip of the right index finger at a rate of $200 \mathrm{~Hz}$.

\section{Data analysis}

Data were pre-processed using custom software written in $\mathrm{R}$ (www.r-project.org). First, data were low-pass filtered at $5 \mathrm{~Hz}$ with a second-order Butterworth filter. Then, reaching trajectories were time-normalized such that each sample represented $1 \%$ of the reaching movements' total time. To ensure that participants did in fact perform a 'default' action during go-on trials, we used an inclusion criterion whereby at least $90 \%$ of all their reaching trajectories in the congruent go trials had to be characterized by a single velocity peak. Because go-on trials and stop trials yielded qualitatively different movements (see Fig. 2 for sample velocity profiles) and did not contain equal trial numbers, they were analysed separately.

Results

Go-on trials The time taken to perform the default reaching movement was significantly longer when the prime was incongruent $(M=615 \mathrm{~ms})$ compared to when it was congruent $(M=600 \mathrm{~ms} ; t(15)=3.46, p<0.01, d=.24)$. Maximum velocity was also significantly slower for trials containing a stop prime $(M=66.80 \mathrm{~cm} / \mathrm{s})$ compared to a go-on prime $(M=68.13 \mathrm{~cm} / \mathrm{s} ; t(15)=2.63, p<0.05, d=.12)$.

Stop trials The percentage of movements correctly inhibited was higher for congruent stop trials, where a stop prime precedes a stop target $(M=79.14 \%)$ compared to incongruent trials $(M=67.25 \% ; t(15)=4.15, p<0.001, d=.61)$. On trials that were successfully stopped, subjects were faster to terminate the default reaching movement and return to the home button following a stop prime $(M=998.40 \mathrm{~ms})$ compared to a go-on prime $(M=1022 \mathrm{~ms} ; t(15)=3.72, p<0.01, d=.34)$. Moreover, their hand moved a shorter distance in the incorrect 'forward' direction on congruent stop trials $(M=303.24 \mathrm{~mm})$ compared to incongruent stop trials $(M=310.60 \mathrm{~mm}, t(15)=$ 4.99, $p<0.001, d=.45)$.

Prime visibility At the end of the experiment, participants completed 5 blocks of a prime detection task. First, participants were shown a normal experimental trial that consisted of the same trial sequence used in the main experiment. However, to eliminate confusion participants were no longer required to respond to the target. Two primes were subsequently shown, one on the left of the screen and one on the right. Participants were asked to indicate which one had appeared as the prime on the previous trial by making a right/left button press. Each trial contained one 'signal location' and one 'noise location'. Responses were scored as hits when the signal location was chosen and as false alarms when the noise location was chosen. Subjectively, none of the participants reported having seen any of the primes. Subliminality of the masked primes was confirmed insofar as the hit rate did not differ from chance (mean hit rate, $50.81 \%, p=0.49)$, and $d$ ' did not differ from zero $(M=0.04$, $p=0.48)$. We also note that, while there was a numerical difference between the $d$ ' values observed in the congruent and incongruent conditions ( $M=0.17, M=0.04$, respectively), this difference was not reliable $(p=0.22)$. 
A

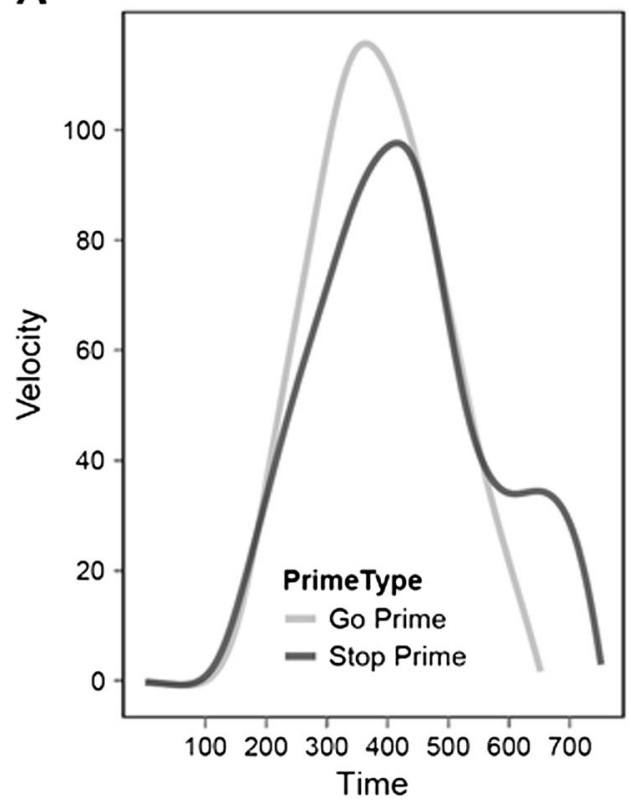

Fig. 2 Example velocity profiles of the default (go-on) reaching movement in the congruent condition (light gray) and incongruent condition (dark gray) in Experiment 1 (a) and Experiment 2 (b). Note

\section{Experiment 2}

In Experiment 2, all experimental procedures remained the same, except for the inclusion of a neutral prime (' $x$ ') on onethird of the trials, which served two purposes. First, a possible explanation of our results in Experiment 1 was that the differences we observed between congruent and incongruent trials were carried in large part by the congruent prime, not the incongruent prime. If that were the case, then the nature of the prime-induced modulation would have more to do with the confirmation of an ongoing action as opposed to the interruption of that action. Because the diagnostic feature of cognitive control is generally taken to be the suppression, or interruption, of an ongoing action, we sought to clarify the direction of the effects we observed in Experiment 1 by including a neutral condition. Second, one might be concerned that our measure of prime visibility was flawed in Experiment 1 because the primes and targets differed only in their physical appearance (i.e., upper-case targets vs. lower-case primes). By including a neutral prime in Experiment 2, we can assess subjects' prime visibility with a stimulus that shares no features with the consciously perceived targets. We note that 16 new participants were recruited for Experiment 2, which now included Prime-target Congruency as a factor with three levels (congruent, incongruent and neutral). We analyzed the continuous dependent variables in Experiment 2 with a one-way ANOVA with Greenhouse-Geisser corrections, and for the accuracy rates we used a generalized linear model with a binomial error distribution and a logit link function.
B

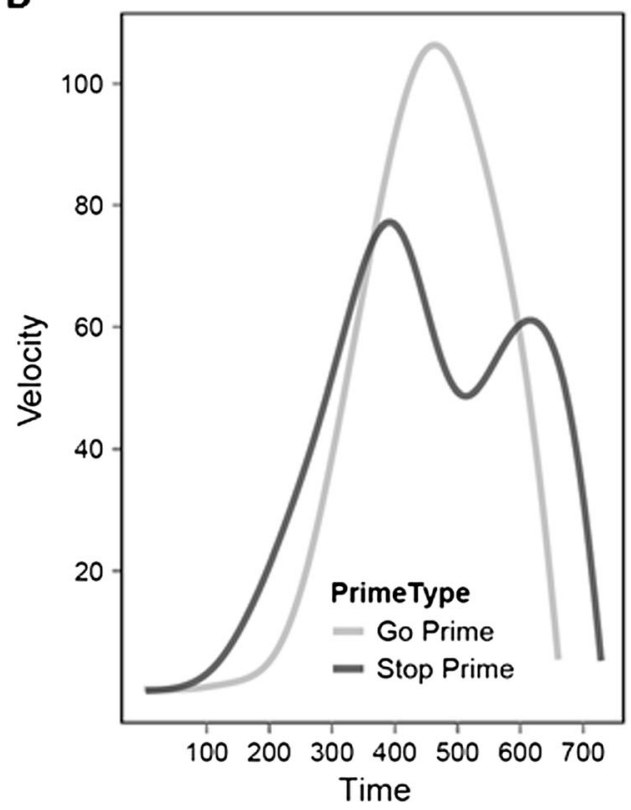

the disruption produced by the stop prime, indicated by the reduced overall velocity $(\mathbf{a}, \mathbf{b})$ and the multiple peaks (b)

Results

Go-on trials There was no main effect of Congruency on movement time $(p>0.05)$ but there was in the analysis of maximum tangential velocity. Here, we found a significant main effect of Congruency, $F(2,30)=3.60, p<0.05, \eta_{\mathrm{p}}{ }^{2}=.25$, such that participants were significantly slower on incongruent go-on trials $(M=66.84 \mathrm{~cm} / \mathrm{s})$ compared with both congruent go-on trials $(M=67.48 \mathrm{~cm} / \mathrm{s} ; t(15)=2.34, p<.05$, $d=.07)$ and neutral go-on trials $(M=67.53 \mathrm{~cm} / \mathrm{s}, t(15)=2.25$, $p<.05, d=.07)$. No differences in maximum velocity were observed between congruent and neutral go-on trials, suggesting that the direction of the effect is due to interference, not facilitation.

Stop trials Accuracy rates were reliably modulated by Congruency $\left(X^{2}(2)=22.1, p<0.0001\right)$. Compared with incongruent stop trials $(M=71.15 \%)$, where go-on primes preceded stop targets, the log odds of correctly inhibiting the ongoing action increased by a ratio of $0.64(p<0.0001)$ during congruent stop trials $(M=82.22 \%)$, and a ratio $0.52(p<0.001)$ during neutral stop trials $(M=80.51 \%)$. The difference in accuracy rates between congruent stop trials and neutral stop trials was not reliable, again suggesting an effect of interference by the incongruent prime as opposed to facilitation from the congruent prime. Congruency also modulated the total distance travelled in the incorrect forward direction during stop trials $\left(F(2,30)=12.29, p<0.001, \eta_{\mathrm{p}}{ }^{2}=.45\right)$. Specifically, participants terminated their forward movement 
sooner on congruent stop trials $(M=270.31 \mathrm{~mm})$ and neutral stop trials $(M=271.32 \mathrm{~mm})$ compared to incongruent stop trials $(M=278.29 \mathrm{~mm})$. The difference between congruent and neutral was not reliable, but the difference between congruent and incongruent was $(t(15)=3.73, p<0.01, d=.34)$ as was the difference between neutral and incongruent $(t(15)=3.59, p<0.01, d=.31)$. Once again, this pattern suggests interference by the incongruent prime as opposed to facilitation by the congruent prime.

Prime visibility In Experiment 2, the neutral prime ' $\mathrm{x}$ ' was also included in the prime detection trials. Unlike the congruent and incongruent primes, the neutral prime never appeared as a target in the main experiment. This eliminated the potential effects of phonological and/or response-based interference, and provided an impartial measure of prime awareness. Once again, none of the participants reported awareness of the primes. Prime visibility calculated across all primes was at chance (hit rate $=52.2 \%, p=0.11$ ) and $d$ ' did not differ significantly from $0(M=0.11, p=0.11)$. Importantly, analyses conducted exclusively over the neutral prime condition revealed that $d$ ' values were not significantly different from 0 $(M=0.17, p=0.25)$.

\section{Discussion}

The present study establishes three important findings. First, default 'go-on' reaching movements are systematically modulated by the mid-flight presentation of a masked (subliminal) stimulus. Specifically, the mid-flight presentation of a masked go-on prime led to faster reaching movements compared to movements that were made in the context of masked stop primes. This result was observed in analyses of both reaching velocity and reaching duration. Thus, while the modulation of default movements by masked primes is small in magnitude, it is robust, having been replicated in Experiments 1 and 2 .

The second important finding is that the mid-flight presentation of a masked stimulus also led to systematic modulations of the 'stop' trials in which the default reaching movement had to be terminated. On these trials, subjects terminated the forward movement and returned to the start position sooner when the masked prime was a congruent stop prime. Similarly, subjects reached further in the (wrong) forward direction in the context of an incongruent go-on prime.

The third important finding is that the direction of the masked congruence effect is carried by the incongruent condition. That is, the mid-flight presentation of a stop prime led to default reaching movements that were slower than the movements performed in the context of both go-on and neutral primes. Similarly, on stop trials, subjects were less successful in terminating their responses following an incongruent go-on prime relative to both neutral and congruent primes.

Taken together, our findings establish that a masked (subliminal) prime stimulus that is presented during a routine behavior can lead to clear and straightforward modulations of that behavior. Furthermore, the results of Experiment 2 demonstrate that these modulatory effects are due to interference from the incongruent prime. Because the diagnostic feature of cognitive control is the interruption of an ongoing action, these results strongly suggest that individuals are capable of non-conscious cognitive control.

This conclusion is further supported by an additional analysis in which we estimated the number of discrete movement segments comprising subjects' overall movements. It is well accepted in the motor-control literature that complex reaching movements are comprised of smaller segments (Flash \& Henis, 1991; Meyer, Abrams, Kornblum, Wright, \& Smith, 1988; Novak, Miller, \& Houk, 2000; Pratt, Chasteen, \& Abrams, 1994). And while the techniques used to identify the individual segments typically begin with an a priori assumption of the shape and amplitude of the velocity profile that individual movement segments may have (e.g., Flash \& Henis, 1991; Krebs, Aisen, Volpe, \& Hogan, 1999), we use here a very simple, empirically-driven technique that delineates an individual movement segment through the identification of local maxima and minima within the tangential velocity profile of the reaching movement. The endpoints of each segment were defined as either movement onset/offset or as local minima between each velocity peak. Critically, if the non-conscious perception of a masked stop prime produces interference in an ongoing action, then trials with multiple movement segments should occur more often on incongruent go-on trials. To investigate this possibility, we pooled the go-on trials across Experiments 1 and 2 and the results confirmed our prediction. Namely, the likelihood that subjects' reaching movements were comprised of multiple movement segments was greater following an incongruent stop prime $(M=12.98 \%)$ than it was following a congruent go-on prime $(M=9.70 \% ; t(31)=2.82, p<$ $0.01, d=.50)$. This finding is best depicted in Fig. 2b. In this case, the perception of the stop prime led to a termination of the default movement (characterized by a sharp decrease in tangential velocity at the midway point), followed by a second movement segment in which the hand continues on its original path (characterized by a second velocity peak). Crucially, the finding that movement hesitations were more likely to occur on trials with incongruent stop primes lends further support to the claim that ongoing actions can be interrupted by a non-consciously perceived stimulus (i.e., non-conscious cognitive control).

In conclusion, our findings demonstrate that the cognitive mechanisms required to monitor and control an ongoing action are continuously being updated and that they are sensitive 
to both consciously and non-consciously perceived information. Our findings extend those of van Gaal and colleagues (2009, 2010a, b) and Hughes and colleagues (2009) who showed that non-consciously perceived stimuli associated with a no-go response interfered with the preparation of an upcoming action. Our findings also extend those from the literature on nonconscious visuomotor control by showing that the real-time control of a routine action can be informed by non-spatial stimulus attributes.

\section{References}

Baars, B. J. (2002). The conscious access hypothesis: Origins and recent evidence. Trends in Cognitive Sciences, 6(1), 47-52.

Dehaene, S., \& Naccache, L. (2001). Towards a cognitive neuroscience of consciousness: Basic evidence and a workspace framework. Cognition, 79(1-2), 1-37.

Finkbeiner, M., Coltheart, M., \& Coltheart, V. (2013). Pointing the way to new contraints on the dynamical claims of computational models. Journal of Experimental Psychology. Human Perception and Performance, 40(1), 172-185.

Flash, T., \& Henis, E. (1991). Arm trajectory modifications during reaching towards visual targets. Journal of Cognitive Neuroscience, 3(3), 220-230.

Gaveau, V., Pisella, L., Priot, A. E., Fukui, T., Rossetti, Y., Pelisson, D., \& Prablanc, C. (2014). Automatic online control of motor adjustments in reaching and grasping. Neuropsychologia, 55, 25-40. doi:10. 1016/j.neuropsychologia.2013.12.005

Hughes, G., Velmans, M., \& De Fockert, J. (2009). Unconscious priming of a no-go response. Psychophysiology, 46(6), 1258-1269. doi:10. 1111/j.1469-8986.2009.00873.x

Krebs, H. I., Aisen, M. L., Volpe, B. T., \& Hogan, N. (1999). Quantization of continuous arm movements in humans with brain injury. Proceedings of the National Academy of Sciences, 96(8), 4645-4649.

Meyer, D. E., Abrams, R. A., Kornblum, S., Wright, C. E., \& Keith Smith, J. E. (1988). Optimality in human motor performance: Ideal control of rapid aimed movements. Psychological Review, 95(3), 340.

Novak, K. E., Miller, L. E., \& Houk, J. C. (2000). Kinematic properties of rapid hand movements in a knob turning task. Experimental Brain Research, 132(4), 419-433.

Pratt, J., Chasteen, A. L., \& Abrams, R. A. (1994). Rapid aimed limb movements: Age differences and practice effects in component submovements. Psychology and Aging, 9(2), 325.

van Gaal, S., Ridderinkhof, K. R., van den Wildenberg, W. P. M., \& Lamme, V. A. F. (2009). Dissociating consciousness from inhibitory control: Evidence for unconsciously triggered response inhibition in the stop-signal task. Journal of Experimental Psychology-Human Perception and Performance, 35(4), 1129-1139. doi:10.1037/ A0013551

van Gaal, S., Lamme, V. A., \& Ridderinkhof, K. R. (2010a). Unconsciously triggered conflict adaptation. PloS One, 5(7), e11508. doi:10.1371/ journal.pone. 0011508

van Gaal, S., Ridderinkhof, K. R., Scholte, H. S., \& Lamme, V. A. (2010b). Unconscious activation of the prefrontal no-go network. Journal of Neuroscience, 30(11), 4143-4150. doi:10.1523/ JNEUROSCI. 2992-09.2010 